\title{
The Surface-Bounded Exosphere of Mercury
}

\author{
Andrew Potter \\ National Solar Observatory, 950 N. Cherry Avenue, Tucson, Arizona \\ 85726, U.S.A.
}

\section{Background}

Almost thirty years ago, the instruments on the Mariner 10 spacecraft detected traces of hydrogen, helium, and possibly oxygen in the atmosphere of Mercury. There the matter rested until the mid-80's, when emission lines of sodium (Potter \& Morgan 1985) and potassium (Potter \& Morgan 1986) were found in the spectrum of Mercury, resulting from resonance scattering of sunlight by metal atoms in the atmosphere. Searches for other metals in the Mercury atmosphere were fruitless until recently, when an emission line attributed to calcium has been observed (Bida, Killen \& Morgan 2000). The densities of all these species are so low that gas phase collisions are negligible, so that the Mercury atmosphere is an exosphere bounded at its base by the surface of the planet. The fact that the exosphere is bounded by the Mercury surface means that interactions with the surface must occur. Energy exchange, surface absorption and desorption, and ion neutralization can take place, and these must be accounted for in models of the exosphere. None of these atmospheric species can survive long on Mercury, being lost mainly by photoionization followed by trapping in the solar wind and partly by solar radiation acceleration. Consequently, the species that we observe must be in a steady state, being generated as fast as they are lost to space. The metals must originate from the surface of the regolith, with the supply of fresh surface material maintained by gardening of the surface by meteoroid impact. The metals are released from the surface into the exosphere by photon-stimulated desorption (Madey et al. 1998), particle sputtering (McGrath, Johnson \& Lanzerotti 1986), and meteoroid impact vaporization (Morgan, Zook \& Potter 1988). Thermal evaporation of species condensed on the cold side may be important for determining their distribution over the planet (Hunten \& Sprague 2002). The interaction and relative importance of all these processes has been discussed by Killen \& Morgan (1993). Much can be said about these processes, but this review is focused primarily on the current status of observations of sodium, potassium, and calcium.

\section{Sodium}

Since the sodium resonance emissions were discovered, hundreds of observations have been made (for example, Sprague, et al. 1997). The average column density of sodium is of the order of $10^{11}$ atoms $/ \mathrm{cm}^{2}$, varying in an apparently random fashion by a factor of two or three throughout the Mercury year, with a tendency to be largest at perihelion and aphelion, and smallest in the parts of the orbit where heliocentric velocity and the corresponding radiation pressure on sodium 
are the largest. The density of sodium is usually not uniform over the planet, displaying "spots" or peaks of sodium emission in north or south hemispheres, or sometimes in both (Potter \& Morgan 1990). These features could result from local concentrations of sodium in the surface rocks (Sprague, Schmitt \& Fink 1998), or evaporation of sodium resulting from implantation of magnetospheric sodium ions in the surface (Sprague 1992, Ip 1993), or directly from sputtering of sodium from the surface rocks (Potter \& Morgan 1990). Another characteristic of the sodium distribution is that it is variable, sometimes changing from day to day (Potter \& Morgan 1990; Potter, Killen \& Morgan 1999). Again, this could be the result of geographic factors or the result of changes in local solar weather, causing either changes in the magnetospheric particle deposition, or variations in the amount of solar particle sputtering. The temperature of the sodium atoms is approximately $1000^{\circ} \mathrm{K}$ (Killen et al. 1999), in reasonable agreement with the temperature expected for sodium released from the surface by photo-stimulated desorption, suggesting that this is the dominant process for sodium production. Sodium column density near the dawn terminator is often greater than at the dusk terminator, suggesting the evaporation of condensed sodium from the cold dark side as it rotates into sunlight (Hunten \& Sprague 2002). Further advances in understanding the processes at work in the sodium exosphere of Mercury await observations at improved spatial resolution. These could be achieved with modern adaptive optics technology.

One of the more intriguing predictions of the models of the sodium exosphere was the prediction that there might be a sodium tail, not unlike a comet tail. If sodium atoms are released from the surface with velocities greater than about $2 \mathrm{~km} / \mathrm{s}$ when Mercury is at greatest heliocentric velocity and maximum radiation pressure, the atoms will escape the planet to form a tail. Ip (1986) and Smyth \& Marconi (1995) both predicted the formation of a tail, and generated models of what the tail might look like. The tail is faint, but can be detected when Mercury is visible against a dark sky just before sunrise or just after sunset (Potter, Killen \& Morgan 2002). Sodium atoms in the tail display velocities up to $4 \mathrm{~km} / \mathrm{s}$ normal to the tail direction, suggesting that an energetic process such as sputtering produces the sodium in the tail. Somewhere between 1 and 10 percent of the sodium generated can be lost to space from the tail. But this happens only when radiation acceleration is high. When it is not, the tail must vanish, or rather turn into an extended cloud around Mercury (Smyth \& Marconi 1995).

Recent modeling studies have improved our understanding of the sodium exosphere. The magnetosphere opens in response to a southward IMF, allowing solar wind particles to impact the surface, leading to sputtering of sodium from the surface (Sarantos et al. 2001). This effect was used to explain changes observed in the sodium exosphere (Killen et al. 2001). The effects of a Solar Energetic Particle event on Mercury have been modeled, and the results show that sodium ions would be precipitated to the surface in patterns similar to those observed (Leblanc et al. 2003). A 3-D Monte Carlo model with all known sources and sinks for sodium has been developed, that reproduces most of the observed features of the sodium exosphere; dawn-dusk asymmetries, high latitude spots, and the sodium tail (Leblanc \& Johnson 2003). 


\section{Potassium}

Potassium is much more difficult to observe than sodium. The $\mathrm{D}_{2}$ line at 7664 A (the strongest emission of the $D_{1}-D_{2}$ pair) is usually hidden under an atmospheric oxygen absorption line, and can be seen only when the Doppler shift is large enough blue-wards to bring it out from under the line. The weaker $\mathrm{D}_{1}$ line at $7699 \mathrm{~A}$ can only be clearly seen when the Doppler shift is large enough to bring it entirely up and out of the potassium Fraunhofer absorption line. So observations of the potassium emission are few. Same-day observations of sodium and potassium show their distributions to be similar, both showing high latitude emission peaks (Potter \& Morgan 1997). Considering the chemical similarity of sodium and potassium, this is not surprising. However, there is a puzzle, in that the ratio of sodium to potassium emission is variable from one observation to the next, with values ranging from about 20 to 140 (Potter et al. 2002). This is in contrast to the Moon, where the $\mathrm{Na} / \mathrm{K}$ ratio stays at about 7 , not far from the ratio of the elements in the surface rocks. One possibility suggested was that potassium levels in the surface rocks vary from place to place (Sprague, Kozlowski \& Hunten 1990) But it appears more likely that some process, as yet not understood, removes potassium faster than sodium from the exosphere, at varying rates (Potter et al. 2002). The magnetosphere may play a role, since it is present on Mercury, but absent on the Moon, where the ratio is closer to the expected value.

\section{Calcium}

Calcium is the most recently discovered element in the Mercury exosphere (Bida, Killen \& Morgan 2000). An emission corresponding to the calcium Ca I line at 4226.7 A was found in spectra taken at evening twilight with the Keck telescope. The line is Doppler-shifted to the blue, corresponding to velocities up to $4 \mathrm{~km} / \mathrm{s}$. The emission line is not seen on the planet itself, but appears at distances up to a planetary diameter away from the planet. The emission is faint, and perhaps that is why no observations are reported close to the planet. On the planet, the weak line would be overwhelmed by stronger reflections from the planetary surface. The source process for the calcium is not understood. It must either be particle sputtering or meteoroid impact since these are the only processes that could generate the observed velocities.

\section{Summary}

Sodium is easy to observe, and so we have many sodium observations. These have stimulated serious theoretical modeling, and we now understand in principle the sodium exosphere. For further refinements, we need measurements with much better spatial resolution, and we need accurate information about the magnetosphere and how it responds to solar influences. Potassium and calcium are less well understood. The measurements are difficult and as a consequence, there is much less information available for these species than for sodium. We realize that there must be still other exospheric species that cannot be detected by ground-based observations. The currently planned missions to Mercury will 
detect these species, and map the magnetosphere, leading to a major improvement in our understanding of the Mercury exosphere, as well as the interaction of the space environment with planetary surfaces.

Acknowledgments. This work was supported by the NASA Planetary Astronomy Program and by the National Solar Observatory.

\section{References}

Bida, T.A., Killen, R.M. \& Morgan, T.H. 2000, Nature, 404, 159

Hunten, D.M. \& Sprague, A.L. 2002, Meteoritics and Planetary Science, 37, 1191

Ip, W. -H. 1986, Geophys. Res. Lett., 13, 423

Ip, W. -H. 1987, Geophys. Res, Lett., 14, 1191

Ip, W. -H. 1993, Ap. J., 418, 451

Killen, R.M. and Morgan, T.H. 1993, Icarus, 101, 293

Killen, R.M., Potter, A.E., Fitzsimmons, A. \& Morgan, T.H. 1999, Planetary \& Space Science, 47, 1449

Killen, R.M., Potter, A.E., Reiff, P., Sarantos, M., Jackson, B.V., Hick, P. \& Giles, B. 2001, Jour. Geophys. Res., 106, 20509

Leblanc, F., Luhmann, J.G., Johnson, R.E. \& Liu, M. 2003, Planetary \& Space Science, 51, 339

Leblanc, F. \& Johnson, R.E. 2003, Icarus, 164, 261

McGrath, M.A., Johnson, R.E., \& Lanzerotti, L.J. 1986, Nature, 323, 694

Madey, T.E., Yakshinskiy, B.V., Ageev, V.N. \& Johnson, R.E. 1998, Jour. Geophys. Res., 103, 58773

Morgan, T.H., Zook, H.A., \& Potter, A.E. 1988, Icarus, 75, 156

Potter, A.E. \& Morgan, T.H. 1985, Science, 229, 651

Potter, A.E. \& Morgan, T.H. 1986, Icarus, 67, 336

Potter, A.E. \& Morgan, T.H. 1990, Science, 248, 835

Potter, A.E. \& Morgan, T.H. 1997, Planetary \& Space Science, 45, 95

Potter, A.E., Killen, R.M. \& Morgan, T.H. 1999, Planetary \& Space Science, 47, 1441

Potter, A.E., Killen, R.M., \& Morgan, T.H. 2002, Meteoritics \& Planetary Science, 37, 1165

Potter, A.E., Anderson, C.M., Killen, R.M., \& Morgan, T.H. 2002, Jour. Geophys. Res., 107, 10.1029/2000Je001493

Sarantos, M., Reiff, P.H., Hill, T.W., Killen, R.M., \& Urquhart, A.L. 2001, PASP, 49, 1629

Smyth, W.H. \& Marconi, M.L. 1995, Ap. J., 441, 839

Sprague, A.L., Kozlowski, R.W.H., \& Hunten, D.M. 1990, Science, 249, 1140

Sprague, A.L. 1992, Jour. Geophys. Res., 97, 18257

Sprague, A.L., Kozlowski, R.W.H., Hunten, D.M., Schneider, N.M., Domingue, D.L., Wells, W.K., Schmitt, W. \& Fink, U. 1997, Icarus, 129, 506

Sprague, A.L., Schmitt, W.J. \& Hill, R.E. 1998, Icarus, 136, 104 